\title{
Innovations of Photodynamic Therapy for Brain Tumors: Potential of Multifunctional Nanoparticles
}

\author{
Denise Bechet ${ }^{1}$, Céline Frochot ${ }^{2,4}$, Regis Vanderesse ${ }^{3,4}$ and Muriel Barberi-Heyob ${ }^{1,4 *}$ \\ ${ }^{1}$ CRAN, Centre de Recherche en Automatique de Nancy, UMR 7039 CNRS, Lorraine-University, Centre Alexis Vautrin, Brabois, Vandoeuvre-lès-Nancy, France \\ ${ }^{2}$ LRGP, Laboratoire Réactions et Génie des Procédés, UPR 3349 CNRS, Lorraine-University, Nancy, France \\ ${ }^{3}$ LCMP, Laboratoire de Chimie-Physique Macromoléculaire, UMR 7568 CNRS, Lorraine-University, Nancy \\ ${ }^{4}$ GDR 3049 "Médicaments Photoactivables - Photochimiothérapie (PHOTOMED)", France
}

\begin{abstract}
The poor outcome of primary malignant brain tumors is predominantly due to local invasion and recurrence. Multifunctional nanoparticles harbouring various functions including targeting, imaging and treatment have been intensively studied aiming to overcome limitations associated with conventional cancer diagnosis and therapy. Multifunctionality can be engineered into a single nanoplatform to provide tumour-specific detection, treatment, and follow-up. This review summarizes different targeting strategies for construction of multifunctional nanoparticles including magnetic nanoparticles-based theranostic systems, and the various surface engineering strategies of nanoparticles for in vivo applications. Using nanoparticles as carriers of photoactivable compounds is a very promising approach as they can satisfy all the requirements for an ideal photodynamic therapy agent. Nanoparticles represent emerging photosensitizer carriers that show great promise for PDT.
\end{abstract}

Keywords: Nanoparticles; Photosensitizer; Photodynamic therapy; Targeting; Imaging; Glioblastoma

Abbreviations: C11Pc: Zn(II)-phthalocyanine disulphide; F3: Peptide Targeting Surface-Localized Vasculature; F98: Rat Glioma Cell Line; HeLa: Human Malignant Cervical Cells; HER2: Human Epidermal Growth Factor Receptor 2; KB: Human Oropharyngeal Cancer Cell Line; HT-29: Human Colon Adenocarcinoma Cell Line; Ir(III): Iridium-complex-functionalized Fe3O4/SiO2; MDA-MB-231: Human Braest Carcinoma Cell Line; MCF-10A: Normal Human Mammary Epithelial Cells; MCF-7: Human Breast Adenocarcinoma Cell Line; NIR: Near Infrared; JMGC803: Human Gastric Carcinoma Cells; PAA: Polyacrylamide; PEG: PolyEthylene Glycol; PHPP: 2,7,12,18-tetramethyl-3,8-di(1-propoxyethyl)-13,17-bis-(3hydroxypropyl)porphyrin; PpIX: Protoporphyrine IX; RGD: ArginineGlycine-Aspartate Peptide; SKBR-3: Human Braest Carcinoma Cell Line; SW480: Human Colon Adenocarcinoma Cell Line; SCC-7: Murine Squamous Cell Carcinoma Cell Line; TSPP: Meso-Tetra(4Sulfonatophenyl)Porphyrin Dihydrochloride

\section{Introduction}

Multifunctional nanoparticles have been developed for disease imaging via passive targeting, but recent advances in nanotechnology have enabled cellular-specific targeting, drug delivery and multi-modal imaging using these nanoplatforms [1]. The goal of this interdisciplinary research is to develop targeted multifunctional nanoparticles, serving as both imaging agents and drug carriers that can effectively pass biological barriers, for diagnosis, staging and treatment of tumors such as brain tumors. Malignant brain tumors are the second most common cause of cancer deaths among youth's people and the sixth most common causes of productive-years loss. Despite advances in neurosurgery and radiotherapy the prognosis for patients with malignant gliomas has changed little for the last decades. Standard treatment of high-grade gliomas usually consists of cytoredictive surgery followed by radiation techniques and chemotherapy.

Photodynamic therapy (PDT) appears to be an interesting modality to fill the need for a targeted treatment that may reduce recurrence and extend survival with minimal side effects. PDT relies on the selective uptake of a photosensitizing molecule in a tumor relative to the surrounding normal parenchyma followed by exposure to the appropriate wavelength of light to activate the photosensitizer. When activated by light irradiation, the photosensitizer interacts with molecular oxygen to produce reactive oxygen species (ROS) and a cytotoxic, short-lived species known as singlet oxygen. Several photosensitizers have been tested in vivo models in brain cancers, demonstrating that using different photosensitizers resulted in varying degree of damage. Interestingly, for example PDT in combination with 5-amino-levulinic acid-induced protoporphyrin IX (ALAPPIX) appears to damage experimental brain tumors selectively, with negligible damage to normal or peripheral edematous tissue [2]. In another study reported by George et al. [3] the authors implanted U87 cells into the brain parenchyma of nude rats and subsequently administered phthalocyanine (Pc 4) and irradiated light to determine: (i) the kinetics of clearance of the drug from the plasma compartment, (ii) the selectivity for glioma versus normal brain tissue, (iii) the possibility of inducing tumor necrosis and/or apoptosis by Pc4mediated PDT [3]. Tumor-specific apoptosis was most uniformly seen; however, necrosis was found mostly in the high-light-dose group. Pc 4 concentration in bulk tumor averaged 3.8 times greater than in normal brain. Namatame et al. [4] evaluated histological changes in a

*Corresponding author: Barberi-Heyob M, CRAN, Research Center for Automatic Control of Nancy, UMR 7039 CNRS, Lorraine-University, Centre Alexis Vautrin, Brabois, Vandoeuvre-lès-Nancy, France, Tel: 33-3-83-59-83-76; Fax: 33-3-83-5983-78; E-mail: m.barberi@nancy.unicancer.fr

Received December 21, 2011; Accepted January 10, 2012; Published January 13, 2012

Citation: Bechet D, Frochot C, Vanderesse R, Barberi-Heyob M (2012) Innovations of Photodynamic Therapy for Brain Tumors: Potential of Multifunctional Nanoparticles. J Carcinogene Mutagene S8:001. doi:10.4172/2157-2518.S8-001

Copyright: (C) 2012 Bechet $\mathrm{D}$, et al. This is an open-access article distributed under the terms of the Creative Commons Attribution License, which permits unrestricted use, distribution, and reproduction in any medium, provided the original author and source are credited. 
rat C6 glioma model treated with a combination of talaporfin sodium, a water-soluble photosensitizer derived from chlorophyll and exposure to a diode laser. PDT was performed to the craniotomized area [4]. Distribution and PDT efficiency of SIM01, a promising photosensitizer, were evaluated on orthotopic C6 tumor model in rats by comparison with Photofrin ${ }^{\circ}$ and $m$ THPC [5]. Pharmacokinetic studies were performed together with ROS determination assays. The correlation between fluorescence and ROS dosimetry appeared conclusive. The apoptotic response of normal brain and intracranial tumor following PDT mediated by five different photosensitizers (Photofrin ${ }^{\circ}$, ALA, chloroaluminum phthalocyanine AICIPc, Tin Ethyl Etiopurpurin SnET2 and $m$ THPC) was also evaluated at 24 hours post-PDT of brain tumors [6].

In spite of the fact that visible light is safe, this approach is suitable to treat only superficial tumors. Ionizing radiations penetrate much deeper and in that case could offer a big advantage in treating patients with tumors in internal organs. Such concept of using multifunctional nanoparticles to yield electrons and radicals in photodynamic and radiation therapies as well their combination is also reviewed in this article.

\section{Interest of multifunctional nanoparticles for PDT}

In bio-nanotechnology, the development of multifunctional nanoparticles can overcome most of the shortcomings of classic photosensitizers [7-10]. The efficiency of PDT is linked to the formation of ROS, and it is commonly accepted that ${ }^{1} \mathrm{O}_{2}$ is the main cytotoxic species that destroys tumor cells. Indeed, once the photosensitizer has absorbed light, it moves from the ground state excited singlet state, and after intersystem crossing excited triplet state. This triplet state can undergo two kinds of reactions and it is commonly accepted that type II reactions, in which the photosensitizer transfers its energy down to the ground state triplet oxygen to produce ${ }^{1} \mathrm{O}_{2}$ is predominant. ${ }^{1} \mathrm{O}_{2}$ can diffuse through the cytoplasm and in biological membranes. As the efficiency of PDT is attributable to the production of ${ }^{1} \mathrm{O}_{2}$, it is unnecessary to release the loaded photosensitizers, but it is essential that the oxygen can diffuse in and out of the nanoparticles. We relate the advantages of using non-biodegradable nanoparticles especially for their ability to allow the diffusion of ${ }^{1} \mathrm{O}_{2}$ in and out.

Polyacrylamide polymers can be used for the synthesis of such nanoparticles, but most of them are ceramic-based (made of silica) or metallic. Their application in drug delivery has not been fully exploited, even though the synthesis of this kind of nanoparticles has been extensively reported in the literature [11]. Different photosensitizers such as 2-devinyl-2-(1-hexyloxyethyl) pyropheophorbide HPPH [12], methylene blue [13] and fullerene [14] have been encapsulated or covalently linked. For a comparison between polyacrylamide and silicabased nanoparticles, methylene blue was loaded in three different types of sub-200 nm nanoparticles: one polyacrylamide based (diameter of 20-30 nm), two silica-based nanoparticles diameter of $190 \mathrm{~nm}$. In terms of drug loading, the polyacrylamide nanoparticles are hydrophilic but small, resulting as expected in a lower loading of methylene blue compared with the loading of the two larger silica-based nanoparticles [13]. The preliminary PDT experiments they performed in vitro (C6 rat glioma cell line) using methylene-blue-loaded polyacrylamide nanoparticles revealed significant photocytotoxicity assessed by confocal microscopy. Calcein acetoxy-methyl ester was used to determine cell viability meanwhile propidium iodide marked dead or dying cells. Incubation was not necessary for the nanoparticles because they were not designed to internalize into target cells but to deliver ${ }^{1} \mathrm{O}_{2}$, thus requiring only external contact with the cell membrane. The nanoparticle matrix protects the entrapped photosensitizer from the biological environment.

Destruction of the vasculature may indirectly lead to tumor eradication, following deprivation of life-sustaining nutrients and oxygen, and this effect is thought to play a major part in the destruction of some tumors by PDT. Hence, tumor vasculature is a potential target of PDT damage. The first example was given by Harrell and Kopelman $[15,16]$. To this end, Kopelman's team synthesized multifunctional platform capable of diagnosis with a MRI contrast enhancer and a photosensitizer, as well as a vector (the integrin-targeting RGD peptide) for specific cell targeting [17-19]. The authors synthesized polyacrylamide nanoparticles containing both the photosensitizer Photofrin ${ }^{\star}$ and MRI contrast-enhancing agents with a surface coating of both polyethylene glycols (PEG) and molecular targeting groups. This assembly enhances the controllable particle residence time owing to the presence of PEG (which inhibits clearance by opsonization) and the recognition of the tumor neovasculature as a result of the targeting groups. In vitro experiments confirmed the production of ${ }^{1} \mathrm{O}_{2}$ at levels believed to be sufficient to cause cell death. In vivo, the MRI contrast agent was useful to monitor changes in tumor diffusion, tumor growth and tumor load. Application of Photofrin ${ }^{\circledR}$-containing nanoparticles followed by irradiation of the photosensitizer produced massive regional necrosis, whereas the tumor cells continued to grow in the control samples $[17,18]$. Applying a similar principle, Reddy and colleagues developed another polyacrylamide nanoparticle encapsulating Photofrin ${ }^{\star}$ and imaging agents (fluorescent dye or iron oxide), this time utilising a F3 peptide targeting surface-localized vasculature [20]. F3 is a 31-amino acid sequence that can accumulate on the cell surface and then translocate to the nucleus of cells of the human breast cancer line MDA-MB-435 both in vitro and in xenograft studies. For this tumor-specific uptake and nuclear localization of F3 peptide, expression of nucleolin, a shuttle protein that traffics between membrane and nucleus located on the cell surface, was a prerequisite. This peptide is a fragment HMGN2 (17-48 amino acid sequence of the $\mathrm{N}$-terminal part of the high human protein 2 ) of the mobility group [21]. Fluorescent probe Alexa Fluor 594 was used to monitor the cellular uptake and subcellular distribution, revealing that the nanoparticles were internalized into the nucleus. In vivo studies demonstrated that the contrast enhancement was increased in both magnitude and duration when targeted nanoparticles were injected in comparison with that of controls that were non-specifically targeted. Sixty days after treatment, $40 \%$ of animals treated with F3-targeted Photofrin nanoparticles were found to be tumor free. Diffusion MRI was used to evaluate changes in tumor diffusion properties as it is known that there is a correlation between the magnitude of diffusion shift and animal survival [22]. It is present in the nuclei of endothelial cells and certain tumor cells such as MDA-MB-435. Several publications of the same team have demonstrated the interest of the use of this targeting peptide coupled to nanoparticles of iron oxide $[23,24]$. A marked advantage of using nanoparticles is that they can serve as multifunctional platforms for brain tumor theranostic. In 2011, Kopelman et al. [17] confirmed in vitro by fluorescence microscopy that nanoparticles functionalized with the peptide F3 was specifically incorporated in the nuclei of 
MDA-MB-435. They demonstrated an improved photocytotoxicity depending on the photosensitizer concentration and the contact time of the targeted nanoparticles. In addition, in vivo these nanoparticles leaded to a high T2 MRI signal intensity at the tumor area. PDT, with the nanoparticles grafted with F3 peptide, highlighted a statistically significant increase in survival over $50 \%$ up to 60 days after treatment [25]. The peptide F3 was also grafted to PAA nanoparticles conjugated with methylene blue $[23,24]$.

Receptors specifically located on angiogenic endothelial cells, such as receptors to vascular endothelial growth factor (VEGF), can be used as molecular targets. Our group has previously described the conjugation of a chlorin (TPC) to a heptapeptide (ATWLPPR), specific for the VEGF receptor, neuropilin-1 (NRP-1) [26,27]. We evidenced a significant decrease in the conjugated photosensitizer cellular uptake after RNA interference-mediated silencing of NRP1 [28]. In vivo, we demonstrated the interest of using this activetargeting strategy, allowing efficient tumour tissue uptake of the conjugated photosensitizer. In mice ectopically xenografted with U87 human malignant glioma cells, we evidenced that only the conjugated photosensitizer allowed a selective accumulation in endothelial cells of tumour vessels. Thanks to an experimental design, an optimal vascular targeted PDT (VTP) condition was selected to show the effects and inter-dependence of photosensitizer dose, fluence and fluence rate on the growth of U87 cells ectopically xenografted in nude mice [29]. Using the peptide-conjugated photosensitizer, induction of tissue factor expression immediately post-treatment, led to the establishment of thrombogenic effects within the vessel lumen [30].

We also described the design and photophysical characteristics of multifunctional nanoparticles consisting of a surface-localized tumor vasculature targeting peptide (ATWLPPR) and encapsulated PDT (Chlorin) and imaging agents (gadolinium oxyde for RMI). Briefly, nanoparticles functionalized with $\sim 4.2$ peptides bound to recombinant neuropilin-1 protein and conferred photosensitivity to cells overexpressing neuropilin-1 [31]. The interest of these multifunctional silica-based nanoparticles was reported [1]. We previously described the photodynamic efficiency of multifunctional silica-based nanoparticles for PDT and the quantification of the ATWLPPR peptide moiety on the nanoparticle surface. Non-biodegradable nanoparticles seemed to be very promising careers satisfying all the requirements for an ideal targeted PDT [31].

Some of the following studies do not directly concern brain tumor models however the used nanoparticles appear interesting for this particular application. Concerning ceramic nanoparticles for PDT, Prasad and colleagues [12] encapsulated 2-divinyl-2-(1- hexyloxyethyl) pyropheophorbide (HPPH), a photosensitizer which is undergoing phase I and II clinical trials for esophageal cancer [32], by controlled hydrolysis of triethylvinylsilane in micellar media. Generation of ${ }^{1} \mathrm{O}_{2}$ by a chemical method and direct luminescence at $1270 \mathrm{~nm}$ was observed and was very similar for HPPH-containing micelles and $\mathrm{HPPH}$ nanoparticles. In vitro experiments (human epithelial ovarian carcinoma UCl-107 and cervical carcinoma Hela cels) showed significant levels of cell death for both HPPH-Tween-80 micelles and HPPH-nanoparticles. In order to prevent release of the photosensitizer, at least partially during systemic circulation, the same team elaborated a new formulation of nanoparticles with the photosensitizer 3-iodobenzylpyropheophorbide attached covalently to the silica matrix
[33]. These investigators proved that ${ }^{1} \mathrm{O}_{2}$ was deactivated mainly outside the nanoparticles. Even if these nanoparticles were not tested on glioma cell lines, their potential use to treat brain tumor should be considered. Wieder and colleagues compared silica and gold nanoparticle systems [34]. They speculated that the surface-bound photosensitizer on the gold nanoparticles might present a real advantage over an encapsulated photosensitizer in terms of diffusion of singlet oxygen [34]. In contrast to ceramic-based nanoparticles, metallic nanoparticles can be confined to an extremely small particle size (a few nanometres). They possess an enormous surface area, and it is possible to attach a large number of photosensitizer molecules on the surface and not be confined to the core of the silica-based nanoparticles. Consequently, ${ }^{1} \mathrm{O}_{2}$ can diffuse more easily [35]. More recently, the same team functionalized a gold nanoparticle by coupling to an anti-HER2 [36] antibody. HER2 or Human Epidermal Growth Factor Receptor-2 (EGFR2) belongs to the family of HER transmembrane receptors responsible for the activation of intracellular signaling pathways involved in cell proliferation. Thus, by coupling anti-HER2 antibody to the nanoparticles, the authors demonstrated a selective in vitro targeting on over expressing cell lines (SK-BR-3) or not (MDA-MB-231). Tada et al. [37] described the preparation and characterization of methylene-blue-containing silica-coated magnetic particles [37]. Their nanomaterial comprised silica spheres of diameter $30 \mathrm{~nm}$ containing magnetic particles of diameter $\sim 11 \mathrm{~nm}$ and methylene blue entrapped in the silica matrix. They showed that ${ }^{1} \mathrm{O}_{2}$ generated by the photosensitizer immobilized in the silica matrix diffused through the solution. The quantum yield was lower in the silica-coated magnetic particles, estimated to be $3 \%$ versus $50 \%$ for methylene blue free in acetonitrile. This could be due to scattering by nanoparticles, local sequestration of ${ }^{1} \mathrm{O}_{2}$ and/or an intrinsically lower quantum yield of encapsulated methylene-bluederived ${ }^{1} \mathrm{O}_{2}$. Lai et al. [38] described the synthesis of a multifunctional system based on iridium-complex-functionalized $\mathrm{Fe}_{3} \mathrm{O}_{4} / \mathrm{SiO}_{2}[\mathrm{Ir}(\mathrm{III})]$ core/shell nanoparticles [38]. The Ir(III) complex plays both the role of the photosensitizer and the phosphorescent probe. With this single system, possessing a three-in-one functionality, it is possible to couple MRI imaging with the $\mathrm{Fe}_{3} \mathrm{O}_{4}$ core, phosphorescence imaging and formation of ${ }^{1} \mathrm{O}_{2}$, with the photosensitizer being encapsulated within $\mathrm{SiO}_{2}$. Upon aeration, both the emission intensity and observed lifetime decreased drastically, suggesting a ${ }^{1} \mathrm{O}_{2}$ quenching process, presumably resulting in production of ${ }^{1} \mathrm{O}_{2}$. The cellular uptake (Hela cells) and imaging ability were confirmed by MRI. HeLa cells treated by PDT showed signs of gradual shrinkage. The formation of bubbles and fragmented cytoplasm clearly indicated the deformation of the cellular membrane and the loss of organelles, which provide sufficient evidence of ${ }^{1} \mathrm{O}_{2}$-induced apoptosis. The main drawback was the $366 \mathrm{~nm}$ irradiation, which is not really compatible with good penetration of light into the tissue.

The ability to directly and specifically target tumor cells or tumor vasculature by a therapeutic agent minimizes systemic exposure to drugs and increases the therapeutic index. Another interesting target is the folic acid receptor, specifically expressed at the brain blood barrier [39]. Thus, the bi-functional nanoparticles can become multi-functional if a targeting agent is coupled to their surface (Table 1). For example, folic acid, a metabolic precursor of a coenzyme, tetrahydrofolate, is involved in the synthesis of purine and pyrimidine nucleic bases constituting nucleic acids. Folic acid-grafted to nanoparticles, nano-objects can be recognized selectively by the folic acid receptor $<$ isomer overexpressed on the surface of certain cancer cells [40]. 


\begin{tabular}{|c|c|c|c|c|c|c|c|c|c|}
\hline \multicolumn{5}{|c|}{ Nanoparticles } & \multirow[b]{2}{*}{$\begin{array}{l}\text { Size } \\
(\mathrm{nm})\end{array}$} & \multirow{2}{*}{ 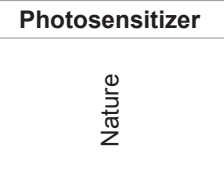 } & \multirow{2}{*}{ 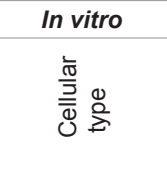 } & \multirow{2}{*}{ 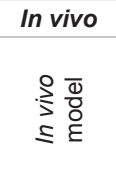 } & \multirow[b]{2}{*}{ 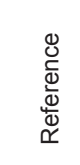 } \\
\hline 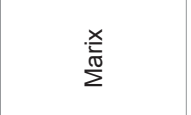 & 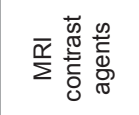 & $\begin{array}{l}\overline{\bar{\Phi}} \\
\frac{\stackrel{c}{\omega}}{}\end{array}$ & 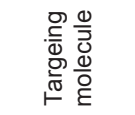 & 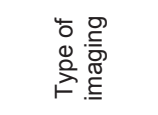 & & & & & \\
\hline Gold & - & PEG & $\begin{array}{c}\text { anti-HER2 } \\
\text { Antibody }\end{array}$ & & 4 & $\mathrm{C} 11 \mathrm{Pc}$ & $\begin{array}{c}\text { MCF-10A } \\
\text { SKBR-3 } \\
\text { MDA-MB-231 }\end{array}$ & & {$[36]$} \\
\hline PAA & - & PEG & F3 Peptide & & - & Methylene blue & MDA-MB-435 & & [23] \\
\hline PAA & - & PEG & F3 Peptide & & 60 & Methylene blue & $\begin{array}{c}\text { 9L } \\
\text { MDA-MB-435 } \\
\text { MCF-7 } \\
\text { F98 }\end{array}$ & & [24] \\
\hline $\begin{array}{l}\text { Chitosane } \\
\text { glycol }\end{array}$ & - & - & & fluorescence & 280 & PpIX & SCC-7 & $\begin{array}{l}\mathrm{HT}-29 \\
\text { mouse }\end{array}$ & {$[60]$} \\
\hline Iron oxyde & Iron oxyde & - & & $\begin{array}{c}\text { MRI } \\
\text { fluorescence }\end{array}$ & 20 & Ce6 & MGC803 & $\begin{array}{l}\text { MGC803 } \\
\text { mouse }\end{array}$ & [61] \\
\hline $\begin{array}{c}\text { Cristaux } \\
\text { (NaYF4, Yb, Er) }\end{array}$ & & PEG & Folic acid & & 30 & Ce6+ doxorubicine & $\begin{array}{l}\text { KB } \\
\text { HeLa }\end{array}$ & & [40] \\
\hline Iron oxyde & Iron oxyde & - & & MRI & 11 & TSPP & & mouse & [62] \\
\hline Chitosan & Iron oxyde & & & MRI & 20 & PHPP & SW480 & $\begin{array}{l}\text { SW480 } \\
\text { mouse }\end{array}$ & [63] \\
\hline Silica & Iron oxyde & & & MRI & $20-30$ & PHPP & SW480 & & [64] \\
\hline Silica & & - & & fluorescence & 105 & Methylene blue & HeLa & $\begin{array}{l}\text { HeLa } \\
\text { mouse }\end{array}$ & [65] \\
\hline PAA & Iron oxyde & PEG & F3 & MRI & 40 & Photofrin ${ }^{\circledR}$ & MDA-MB-435 & $9 \mathrm{~L}$ rat & [25] \\
\hline Silica & Iron oxyde & - & & MRI & 55 & $\operatorname{Ir}(\mathrm{III})$ complex & HeLa & & [38] \\
\hline Silica & Iron oxyde & - & & MRI & 30 & Methylene blue & & & [37] \\
\hline PAA & Iron oxyde & PEG & RGD & & $30-60$ & Photofrin $®$ & $\begin{array}{c}\text { MDA-MB-435 } \\
\text { MCF-7 }\end{array}$ & $9 \mathrm{~L}$ rat & {$[17,18]$} \\
\hline
\end{tabular}

Table 1: Recent studies using multifunctional nanoparticles.

New developments: improvements in the photophysical properties of nanoparticles and their use in combined therapy

The limitation of PDT in brain is extent of light penetration within brain tissue; the following nanoparticles could be an alternative. Twophoton absorption (TPA)-induced excitation of photosensitizers is one of the promising approaches to increase light penetration. Indeed, appropriate photosensitizers can absorb simultaneously two lessenergetic photons; the excitation in the near infra-red (IR) region avoids wasteful tissue absorption or scattering and induces a deeper penetration of light into the tissue. To this end, various molecules with relatively large TPA cross-sections have been designed [41-44]. De Gao et al. [45] described the elaboration of 5,10,15,20-tetrakis(1methyl 4-pyridino)porphyrin tetra( $p$-toluenesulfonate) (TMPyP), encapsulated in polyacrylamide-based nanoparticles. Infrared twophoton nanoplatform phototoxicity was demonstrated in vitro by modulating the time of exposure to light [45]. Kim et al. [46] described the synthesis of organically modified silica nanoparticles co-encapsulating HPPH and an excess of 9,10-bis [4'-(4"-aminostyryl) styryl)anthracene (BDSA)], a highly two-photon-active molecule as a donor [46]. HPPH absorption in nanoparticles had significant overlap with the fluorescence of BDSA aggregates, which enabled an efficient energy transfer through Forster resonance energy transfer (FRET) mechanisms. After indirect two-photon excitation $(850 \mathrm{~nm})$, the authors proved that the energy of the near-IR light was efficiently up-converted (see below) by BDSA aggregates such that it excited $\mathrm{HPPH}$, followed by formation of ${ }^{1} \mathrm{O}_{2}$. The intracellular FRET efficiency was estimated as being approximately $36 \%$. Drastic changes in the morphology of the cells (human cervix epitheloid carcinoma HeLa cells) were observed [46], indicative of impending death.

Zhang et al. [47] described a new concept based on so-called 'photon up-converting nanoparticles' (PUNPs). Up-conversion, in which excitatory light at a certain wavelength produces emission at a shorter wavelength, is very promising as with PUNPs it becomes possible to excite the photosensitizers in the near IR. The PUNPs used are the most efficient photon-up converting phosphors [48]. The kinetics of formation of ${ }^{1} \mathrm{O}_{2}$ is very similar to those observed by Tang et al. and Kopelman [13,17]. A mouse monoclonal antibody against the tumor-associated mucin MUC1 (episialin) was attached covalently to the photosensitizer merocyanine-540-coated PUNPS. After IR irradiation, human breast cancer cells shrunk and a photocytotoxic effect was observed [48].

Another new application with great potential is the use of nanoparticles for a combined therapy in which radiation therapy is used together with PDT in a technique called self-lighting photodynamic therapy (SLPDT). Chen and Zhang have designed a new PDT agent system combining radiation and PDT, in which scintillation luminescent nanoparticles are attached to the photosensitizers [49]. Upon exposure to ionizing radiation, such as X-rays, scintillation luminescence will emit from the nanoparticles and activate the photosensitizers. The advantages of SLPDT compared with radiation therapy or PDT alone are: (i) light delivery to the photosensitizer is 
not necessary; (ii) radiotherapy and PDT are combined and activated by a single source, leading to a simple and less expensive system than PDT alone or both therapies used simultaneously; and (iii) similar therapeutic results can be achieved using lower doses of radiation. Morgan et al. [50] calculated the physical parameters for these nanoparticle conjugates to deliver cytotoxic levels of ${ }^{1} \mathrm{O}_{2}$ at therapeutic radiation doses [50]. They showed that it is necessary to optimize the light yields of nano scintillators, the efficiency of energy transfer to the photosensitizers, the cellular uptake of the nanoparticles to obtain a cytotoxic effect. Even so, the efficacy of the combination therapy will likely be restricted to X-ray energies below $300 \mathrm{keV}$, which limits the application to brachytherapy.

Several studies have shown the potential of this excitation source with nanoparticles coupled to folic acid to target folic acid receptor $[51,52]$.

Concerning brain tumors, a very recent study [53] showed that X-rays could activate Photofrin ${ }^{\oplus}$ from rare-earth formulated particles, leading to a dramatic reduction in the human glioblastoma cells viability. Severe suppression ( $90 \%$ compared to controls) in the metabolic activity of human glioblastoma cells due to the presence of clinically relevant concentration of Photofrin ${ }^{\circ}(20 \mu \mathrm{g} / \mathrm{ml})$, with $\mathrm{Gd} 2 \mathrm{O} 2 \mathrm{~S}$ : $\mathrm{Tb}$ particles $(5 \mathrm{mg} / \mathrm{ml})$ and $120 \mathrm{kVp}$ X-Ray exposure was observed.

Hyperthermia therapy can be used to improve the efficacy of various cancer treatments. For instance, localized hyperthermia can be suggested in the treatment of malignant brain tumors [54]. Hyperthermia treatment for cancer is where the body tissue is exposed to high temperatures. Research has shown that high temperatures can damage and kill cancer cells, usually with minimal injury to normal tissues. In 2004, it was proposed by the National Cancer Institute that by killing cancer cells and damaging proteins and structures within the cells, hyperthermia treatment might shrink tumors. Preliminary data suggest that heat may be especially destructive to two types of tumor cells those that are making deoxyribonucleic acid in preparation for division and those that are acidic and poorly oxygenated. These cell types tend to be resistant to radiation. In this context, the use of inorganic materials to form magnetic nanoparticles for hyperthermia therapy is of great interest for localized treatment of brain cancer without effecting adjacent healthy tissue. Extensive clinical trials have begun using magnetic hyperthermia in animal models (for a recent review, see [55]). $\mathrm{Fe}_{3} \mathrm{O}_{4}$-photosensitizer nanoparticles are of great interest since this system can be used in a combinational treatment with both PDT and hyperthermia therapies [56]. Indeed, hyperthermia can increase the cellular uptake of oxygen molecules [57] that is essential for PDT, and the preferential uptake of porphyrin derivatives in tumors might help target the magnetic nanoparticles to tumors. The crystalline state of the nanoparticles is retained, which assures that the magnetic properties of the excited triplet state are not quenched by the superparamagnetic species in close proximity. Primo et al. [58] reported on the synthesis and characterization of molybdenum chlorophyllin (Mo-Chl) compounds associated in a complex with magnetic nanoparticles (citrate-coated cobalt ferrite), the latter prepared as a biocompatible magnetic fluid. The complex material was developed for application as a synergic drug for cancer treatment using PDT and hyperthermia. Cellular viability was evaluated using gingival fibroblasts cells as a biological model. The study showed that natural photosensitizers molecules Mo-Chl used in association with magnetic nanoparticles represent a promising generation of drug developed to work synergistically in the treatment of neoplastic tissues using PDT and hyperthermia [58].

A very recent study reports on the development of bovin serum albumin nanospheres for synergic application while combining PDT and hyperthermia [59]. The in vitro assay using human fibroblasts revealed no cytotoxic effect demonstrating the potential of the tested system as a synergistic drug delivery system.

\section{Conclusion}

Multifunctional nanoparticles have emerged as a potential vector for brain delivery, able to overcome problems associated with current strategies. Nanoparticles can indeed serve as targeted delivery devices for therapies including PDT, radiotherapy, anti-vascular and thermotherapy. However, the study of nanoparticle-based PDT is still in its infancy, and much remains to be learned. Overall, the studies reported here suggest that this novel modality is promising and might represent a new direction for brain cancer nanotechnology in the near future. Potential clinical use of these multifunctional nanoparticles includes imaging and PDT.

\section{References}

1. Bechet D, Couleaud P, Frochot C, Viriot ML, Guillemin F et al. (2008) Nanoparticles as vehicles for delivery of photodynamic therapy agents. Trends Biotechnol 26: 612-621.

2. Olzowy B, Hundt CS, Stocker S, Bise K, Reulen HJ, et al. (2002) Photoirradiation therapy of experimental malignant glioma with 5-aminolevulinic acid. J Neurosurg 97: 970-976.

3. George JE, Ahmad Y, Varghai D, Li X, Berlin J, et al. (2005) Pc 4 photodynamic therapy of U87-derived human glioma in the nude rat. Lasers Surg Med 36 383-389.

4. Namatame H, Akimoto J, Matsumura H, Haraoka J, Aizawa K (2008) Photodynamic therapy of C6-implanted glioma cells in the rat brain employing second-generation photosensitizer talaporfin sodium. Photodiagnosis Photodyn Ther 5: 198-209.

5. Olivier D, Bourre L, El-Sabbagh E, Loussouarn D, Simonneaux G, et al. (2007) Photodynamic effects of SIM01, a new sensitizer, on experimental brain tumors in rats. Surg Neurol 68: 255-263.

6. Lilge L, Portnoy M, Wilson BC (2000) Apoptosis induced in vivo by photodynamic therapy in normal brain and intracranial tumour tissue. Br J Cancer 83: 11101117.

7. Prasad PN (2004) Polymer science and technology for new generation photonics and biophotonics. Curr Opin Solid State Mater Sci 8: 11-19.

8. Chen W (2006) Nanoparticle based photodynamic therapy for cancer treatment American Physical Society 5-7.

9. Jain KK (2008) Recent advances in nanooncology. Technol Cancer Res Treat 7: $1-13$

10. Allison RR, Mota HC, Bagnato VS, Sibata CH (2008) Bio-nanotechnology and photodynamic therapy-State of the art review. Photodiagnosis Photodyn Ther 5: 19-28.

11. Li H, Marotta DE, Kim S, Busch TM, Wileyto EP, et al. (2005) High payload delivery of optical imaging and photodynamic therapy agents to tumors using phthalocyanine-reconstituted low-density lipoprotein nanoparticles. J Biomed Opt 10: 41203

12. Roy I, Ohulchanskyy TY, Pudavar HE, Bergey EJ, Oseroff AR, et al. (2003) Ceramic-based nanoparticles entrapping water-insoluble photosensitizing anticancer drugs: a novel drug-carrier system for photodynamic therapy. J Am Chem Soc 125: 7860-7865. 
Citation: Bechet D, Frochot C, Vanderesse R, Barberi-Heyob M (2012) Innovations of Photodynamic Therapy for Brain Tumors: Potential of Multifunctional Nanoparticles. J Carcinogene Mutagene S8:001. doi:10.4172/2157-2518.S8-001

Page 6 of 7

13. Tang W, Xu H, Kopelman R, Philbert MA (2005) Photodynamic characterization and in vitro application of methylene blue-containing nanoparticle platforms. Photochem Photobiol 81: 242-249.

14. Davydenko MO, Radchenko EO, Yaschchuk VM, Dmitruk IM, Prylutskyy YI, et al. (2006) Sensibilization of fullerene $C_{60}$ immobilized at silica nanoparticles for cancer photodynamic therapy. J Molecular Liquids 145-147

15. Harrell JA, Kopelman R (2001) Biocopmpatible probes measure intracellular activity. Biophotonics Int 7: 22-24.

16. Xu H, Aylott JW, Kopelman R, Miller TJ, Philbert MA (2001) A real-time ratiometric method for the determination of molecular oxygen inside living cells using sol-gel-based spherical optical nanosensors with applications to rat C6 glioma. Anal Chem 73: 4124-4133.

17. Kopelman R, Koo Y-EL, Philbert M, Moffat BA, Reddy GR, et al. (2005) Multifonctional nanoparticle platforms for in vivo MRI enhancement and photodynamic therapy of a rat brain cancer. J Magn Magn Mat 293: 404-410.

18. Ross B, Rehemtulla A, Koo YE, Reddy GR, Gwangseong K, et al. (2004) Photonic and magnetic nanoexplorers for biomedical use: from subcellular imaging to cancer diagnostics and therapy. Proc of SPIE 5331: 76.

19. Koo YE, Fan W, Hah H, Xu H, Orringer D, et al. (2007) Photonic explorers based on multifunctional nanoplatforms for biosensing and photodynamic therapy. Appl Opt 46: 1924-1930

20. Reddy GR, Bhojani MS, McConville P, Moody J, Moffat BA, et al. (2006) Vascular targeted nanoparticles for imaging and treatment of brain tumors. Clin Cancer Res 12: 6677-6686.

21. Porkka K, Laakkonen P, Hoffman JA, Bernasconi M, Ruoslahti E (2002) A fragment of the HMGN2 protein homes to the nuclei of tumor cells and tumor endothelial cells in vivo. Proc Natl Acad Sci USA 99: 7444-7449.

22. Moffat BA, Chenevert TL, Meyer CR, McKeever PE, Hall DE, et al. (2006) The functional diffusion map: an imaging biomarker for the early prediction of cancer treatment outcome. Neoplasia 8: 259-267.

23. Qin M, Hah HJ, Kim G, Nie G, Lee YE, et al. (2011) Methylene blue covalently loaded polyacrylamide nanoparticles for enhanced tumor-targeted photodynamic therapy. Photochem Photobiol Sci 10: 832-841.

24. Hah HJ, Kim G, Lee YE, Orringer DA, Sagher O, et al. (2011) Methylene blueconjugated hydrogel nanoparticles and tumor-cell targeted photodynamic therapy. Macromol Biosci 11: 90-99.

25. Reddy GR, Bhojani MS, McConville P, Moody J, Moffat BA, et al. (2006) Vascular targeted nanoparticles for imaging and treatment of brain tumors. Clin Cancer Res 12: 6677-6686

26. Thomas N, Bechet D, Becuwe P, Tirand L, Vanderesse R, et al. (2009) Peptideconjugated chlorin-type photosensitizer binds neuropilin-1 in vitro and in vivo. $J$ Photochem Photobiol B 96: 101-108.

27. Tirand L, Frochot C, Vanderesse R, Thomas N, Trinquet E, et al. (2006) A peptide competing with VEGF165 binding on neuropilin-1 mediates targeting of a chlorin-type photosensitizer and potentiates its photodynamic activity in human endothelial cells. J Control Release 111: 153-164.

28. Thomas N, Pernot M, Vanderesse R, Becuwe P, Kamarulzaman E, et al. (2010) Photodynamic therapy targeting neuropilin-1: Interest of pseudopeptides with improved stability properties. Biochem Pharmacol 80: 226-235.

29. Tirand L, Bastogne T, Bechet D, Linder M, Thomas N, et al. (2009) Response surface methodology: an extensive potential to optimize in vivo photodynamic therapy conditions. Int J Radiat Oncol Biol Phys 75: 244-252.

30. Bechet D, Tirand L, Faivre B, Plenat F, Bonnet C, et al. (2010) Neuropilin-1 Targeting Photosensitization-Induced Early Stages of Thrombosis via Tissue Factor Release. Pharm Res 27: 468-479.

31. Couleaud P, Morosini V, Frochot C, Richeter S, Raehm L, et al. (2010) Silicabased nanoparticles for photodynamic therapy applications. Nanoscale 2: 1083-1095.

32. Henderson BW, Bellnier DA, Greco WR, Sharma A, Pandey RK, et al. (1997) $A n$ in vivo quantitative structure-activity relationship for a congeneric series of pyropheophorbide derivatives as photosensitizers for photodynamic therapy Cancer Res 57: 4000-4007.

33. Ohulchanskyy TY, Roy I, Goswami LN, Chen Y, Bergey EJ, et al. (2007) Organically modified silica nanoparticles with covalently incorporated photosensitizer for photodynamic therapy of cancer. Nano Lett 7: 2835-2842.

34. Wieder ME, Hone DC, Cook MJ, Handsley MM, Gavrilovic J, et al. (2006) Intracellular photodynamic therapy with photosensitizer-nanoparticle conjugates: cancer therapy using a 'Trojan horse'. Photochem Photobiol Sci 5: $727-734$.

35. Wang S, Gao R, Zhou F, Selke M (2004) Nanomaterials and Singlet Oxygen Photosensitizers: Potential Applications in Photodynamic Therapy. J Mate Chem 14: 487-493.

36. Stuchinskaya T, Moreno M, Cook MJ, Edwards DR, Russell DA (2011) Targeted photodynamic therapy of breast cancer cells using antibody-phthalocyaninegold nanoparticle conjugates. Photochem Photobiol Sci 10: 822-831.

37. Tada DB, Vono LL, Duarte EL, Itri R, Kiyohara PK, et al. (2007) Methylene blue-containing silica-coated magnetic particles: a potential magnetic carrier for photodynamic therapy. Langmuir 23: 8194-8199.

38. Lai CW, Wang YH, Lai CH, Yang MJ, Chen CY, et al. (2008) Iridiumcomplex-functionalized $\mathrm{Fe} 3 \mathrm{O} 4 / \mathrm{SiO} 2$ core/shell nanoparticles: a facile threein-one system in magnetic resonance imaging, luminescence imaging, and photodynamic therapy. Small 4: 218-224.

39. Wu D, Pardridge WM (1999) Blood-brain barrier transport of reduced folic acid Pharmaceutical Research 16: 415-419.

40. Wang C, Cheng L, Liu Z (2011) Drug delivery with upconversion nanoparticles for multi-functional targeted cancer cell imaging and therapy. Biomaterials 32 1110-1120.

41. Nielsen CB, Johnsen M, Arnbjerg J, Pittelkow M, Mcllroy SP, et al. (2005) Synthesis and Characterization of Water-Soluble Phenylene-Vinylene-Based Singlet Oxygen Sensitizers for Two-Photon Excitation. J Org Chem 70: 70657079 .

42. Oar MA, Serin JM, Dichtel WR, Frechet JMJ Photosensitization of Singlet Oxygen via Two-Photon-Excited Fluorescence Resonance Energy Transfer in a Water-Soluble Dendrimer. Chem Mater 17 2267-2275.

43. Frederiksen PK, Mcllroy SP, Nielsen CB, Nikolajsen L, Skovsen E, et al. (2005) Two-photon photosensitized production of singlet oxygen in water. J Am Chem Soc 127: 255-269.

44. Drobizhev M, Stepanenko Y, Dzenis Y, Karotki A, Rebane A, et al. (2004) Understanding strong two-photon absorption in pi-conjugated porphyrin dimers via double-resonance enhancement in a three-level model. J Am Chem Soc 126: $15352-15353$

45. Gao D, Agayan RR, Xu H, Philbert MA, Kopelman R (2006) Nanoparticles for two-photon photodynamic therapy in living cells. Nano Lett 6: 2383-2386.

46. Kim S, Ohulchanskyy TY, Pudavar HE, Pandey RK, Prasad PN (2007) Organically modified silica nanoparticles co-encapsulating photosensitizing drug and aggregation-enhanced two-photon absorbing fluorescent dye aggregates for two-photon photodynamic therapy J Am Chem Soc 129: 2669 2675

47. Zhang P, Steelant W, Kumar M, Scholfield M (2007) Versatile photosensitizers for photodynamic therapy at infrared excitation. J Am Chem Soc 129: 45264527

48. Heer S, Kompe K, Gudel HU, Haase M (2004) Highly Efficient Multicolour Upconversion Emission in Transparent Colloids of Lanthanide-Doped NaYF4 Nanocrystals. Adv Mater 16: 2102.

49. Chen W, Zhang J (2006) Using nanoparticles to enable simultaneous radiation and photodynamic therapies for cancer treatment $\mathrm{J}$ Nanosci Nanotechnol 6 : 1159-1166.

50. Morgan NY, Kramer-Marek G, Smith PD, Camphausen K, Capala J (2009) Nanoscintillator conjugates as photodynamic therapy-based radiosensitizers: calculation of required physical parameters. Radiat Res 171: 236-244. 
Citation: Bechet D, Frochot C, Vanderesse R, Barberi-Heyob M (2012) Innovations of Photodynamic Therapy for Brain Tumors: Potential of Multifunctional Nanoparticles. J Carcinogene Mutagene S8:001. doi:10.4172/2157-2518.S8-001

51. Chen W (2008) Nanoparticle self-lighting photodynamic therapy for cancer treatment. J Biomed Nanotechnol 4: 369-376.

52. Liu Y, Chen W, Wang S, Joly AG (2008) Investigation of water-soluble x-ray luminescence nanoparticles for photodynamic activation. Appl Phys Lett 92.

53. Abliz E, Collins JE, Bell H, Tata DB (2011) Novel applications of diagnostic $\mathrm{X}$-rays in activating a clinical photodynamic drug: Photofrin II through X-ray induced visible luminescence from "rare-earth" formulated particles. J X-Ray Sci Technol 19: 521-530.

54. Kobayashi T, Tanaka T, Kida Y, Matsui M, Ikeda T (1989) Interstitial hyperthermia of experimental brain tumor using implant heating system. J Neurooncol 7: 201-208

55. Stone R, Willi T, Rosen Y, Mefford OT, Alexis F (2011) Targeted magnetic hyperthermia. Therapeutic Delivery 2: 815-838

56. Gu H, Xu K, Yang Z, Chang CK, Xu B (2005) Synthesis and cellular uptake of porphyrin decorated iron oxide nanoparticles-a potential candidate for bimodal anticancer therapy. Chem Commun 4270-4272.

57. Streffer C, Vaupel P, Hahn GM (1990) Biological Basis of Oncologic Thermotherapy. Berlin Springer 169

58. Primo FL, Cordo PLAG, Neto AF, Morais PC, Tedesco AC (2010) Nanoparticles of molybdenum chlorophyllin photosensitizer and magnetic citrate-coated cobalt ferrite complex available to hyperthermia and photodynamic therapy clinical trials. In AIP Conference Proceedings Rostock 293-297.
59. Simioni AR, Rodrigues MM, Primo FL, Morais PC, Tedesco AC (2011) Effect of diode-laser and AC magnetic field of bovine serum albumin nanospheres loaded with phthalocyanine and magnetic particSles. J nanosci nanotechnol 11: $3604-3608$

60. Lee SJ, Koo H, Lee DE, Min S, Lee S, et al. (2011) Tumor-homing photosensitizer-conjugated glycol chitosan nanoparticles for synchronous photodynamic imaging and therapy based on cellular on/off system. Biomaterials 32: 4021-4029.

61. Huang P, Li Z, Lin J, Yang D, Gao G, et al. (2011) Photosensitizer-conjugated magnetic nanoparticles for in vivo simultaneous magnetofluorescent imaging and targeting therapy. Biomaterials 32: 3447-3458.

62. Ding J, Zhao J, Cheng K, Liu G, Xiu D (2011) In vivo photodynamic therapy and magnetic resonance imaging of cancer by TSPP-coated $\mathrm{Fe} 3 \mathrm{O} 4$ nanoconjugates. J Biomed Nanotechnol 6: 683-686.

63. Sun $Y$, Chen ZL, Yang XX, Huang P, Zhou XP, et al. (2009) Magnetic chitosan nanoparticles as a drug delivery system for targeting photodynamic therapy Nanotechnology 20: 135102

64. Chen ZL, Sun Y, Huang P, Yang XX, Zhou XP (2009) Studies on Preparation of Photosensitizer Loaded Magnetic Silica Nanoparticles and Their Anti-Tumor Effects for Targeting Photodynamic Therapy. Nanoscale Res Lett 4: 400-408.

65. He X, Wu X, Wang K, Shi B, Hai L (2009) Methylene blue-encapsulated phosphonate-terminated silica nanoparticles for simultaneous in vivo imaging and photodynamic therapy. Biomaterials 30: 5601-5609.
This article was originally published in a special issue, Anticancer Drugs handled by Editor(s). Dr. Philippe Becuwe, Henri Poincaré's University of Nancy, France 